\title{
Torsion of term gravid uterus with a large leiomyoma
}

\section{Berna Aslan Çetin*, Pınar Yalçın Bahat, Selin Dikmen, Erkan Şimşek, Alev Aydın}

Department of Perinatology, Kanuni Sultan Süleyman Research and Education Hospital, İstanbul

Received: 01 November 2016

Accepted: 29 November 2016

*Correspondence:

Dr. Berna Aslan Çetin,

E-mail: bernaaslan14@hotmail.com

Copyright: (c) the author(s), publisher and licensee Medip Academy. This is an open-access article distributed under the terms of the Creative Commons Attribution Non-Commercial License, which permits unrestricted non-commercial use, distribution, and reproduction in any medium, provided the original work is properly cited.

\section{ABSTRACT}

Torsion of gravid uterus is a rare obstetric event and it is almost always diagnosed during cesarean section. Leiomyomas and previous cesarean sections are predisposing risk factors. We report a case of 180 degree torsion of a term gravid uterus with only symptom of hematuria.

Keywords: Cesarean section, Posterior hysterotomy, Uterine torsion

\section{INTRODUCTION}

Torsion of a gravid uterus is defined as a more than 45 degree rotation of the uterus along its long axis. It is a relatively rare obstetric event at term. It is almost always diagnosed during cesarean section or laparotomy. ${ }^{1}$ Here we present a case of torsion of a gravid uterus at term and review the literature on predisposing factors, diagnosis and treatment options.

\section{CASE REPORT}

A 29-year-old woman, gravida 4, para 2 and abortus 1 was admitted at 39 weeks' gestation to our emergency department with regular uterine contractions. Her obstetrical history revealed two previous cesarean sections, 15 and 9 years ago. Her $3^{\text {rd }}$ pregnancy failed due to abortion at the $7^{\text {th }}$ week. An ultrasound examination showed a singleton fetus; placenta and a $15 \mathrm{~cm}$ type 6 uterine fibroid were located posteriorly. On vaginal examination cervix was closed and not effaced. Cesarean delivery decision was made due to previous cesarean sections. When Foley catheter was inserted urine was seen bloody. The patient complaint of bloody urine since last one month and she had no other complaints. Urine analysis confirmed blood (3+) in the urine. Cesarean delivery was carried out for previous cesarean sections. Abdominal cavity was opened by Pfannenstiel incision.
Intraoperatively, 180 degree dextratorsion of the uterus was diagnosed. Left round ligament, ovary and tube were rotated to right, lying anteriorly and vessels were extremely engorged (Figure 1). Following unsuccessful attempts at detorsioning the uterus, a posterior low transverse hysterotomy was performed for delivery. A 4300 gr male infant with APGAR scores 9 and 10 at the $1^{\text {st }}$ and $5^{\text {th }}$ minutes, respectively was delivered. The placenta was removed manually. After exteriozation of the uterus out of the abdominal cavity the posterior hysterotomy incision was closed (Figure 2). Following detorsion of the uterus a $15 \mathrm{~cm}$ fibroid on the anterior wall was seen (Figure 3). Then uterus was put back into the abdomen. Analysing the previous reports and ultrasound images in the patient's pregnancy record, we confirmed that a $5 \mathrm{~cm}$ anterior leiomyoma and an anterior placental position had been observed at 18 weeks' scan, exactly the opposite of the posterior position we have seen by ultrasound at the time of hospital admission. Mother and baby made uneventful recoveries. Patient was discharged with her baby on postoperative second day. Ultrasound examination showed a $8 \times 7 \mathrm{~cm}$ fibroid located anteriorly on postoperative $15^{\text {th }}$ day. Urine analysis revealed no blood in the urine. Patient came to outpatient clinic on postoperative 40 th day, a posterior hysterotomy scar and a $6 \times 6 \mathrm{~cm}$ fibroid on the anterior surface were seen on transvaginal ultrasound examination. Patient had no complaints. 


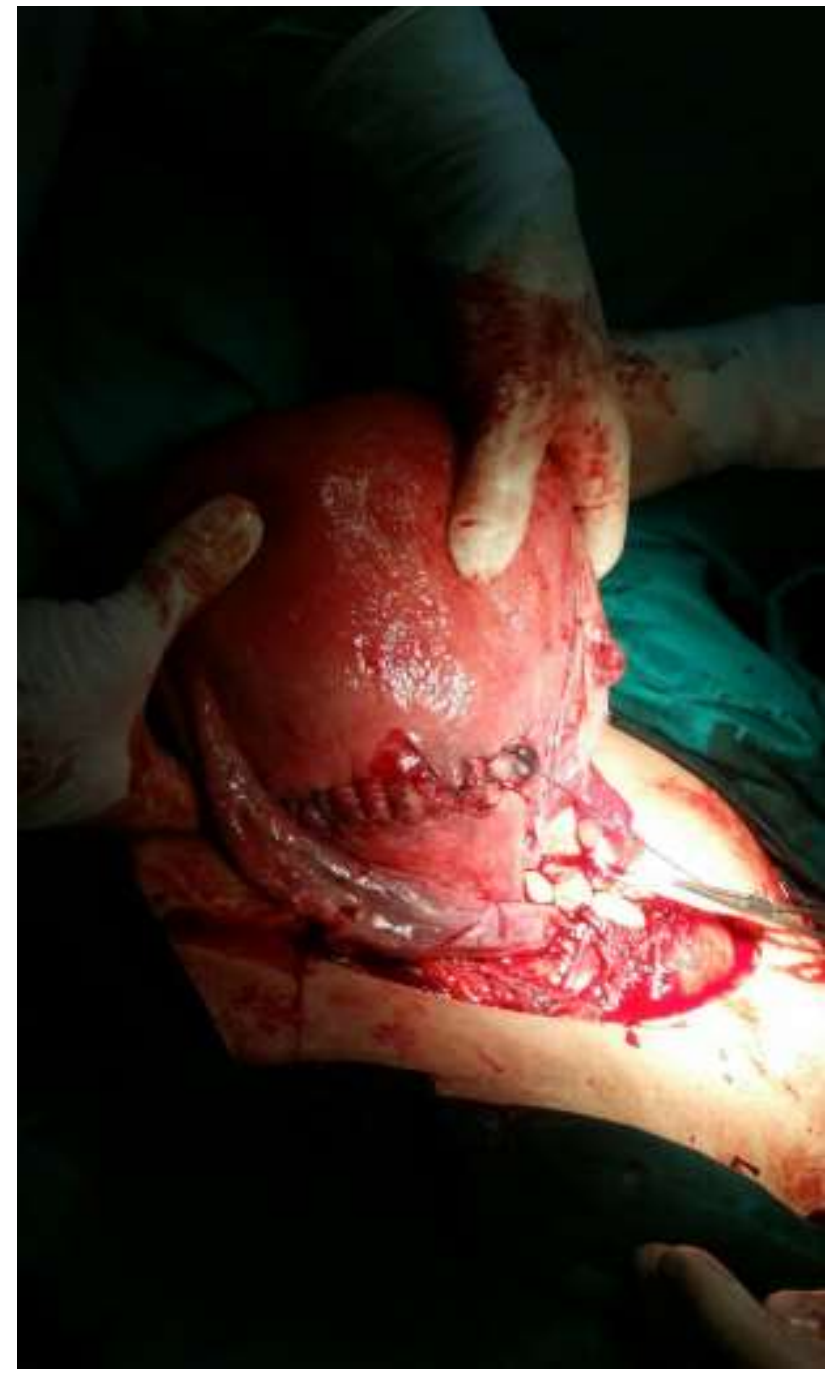

Figure 1: Anterior view before detorsion.

\section{DISCUSSION}

Uterine torsion in pregnancy is defined as a rotation of the uterus on its long axis more than 45 degrees and is an unusual condition that has been referred to as 'once in a lifetime diagnosis'. 2 There are only case reports, no reported cohorts of uterine torsions exist in the literature. This condition can occur in all age groups of the reproductive period, all parity, and all stages of pregnancy. Dextrorotation occurs in two thirds of these cases and levorotation in rest of the one third cases. ${ }^{3}$

It is difficult to study the real epidemiology of this condition due to its rarity. Although it is not possible to find a clear etiology, myomas, uterine malformations, pelvic adhesions, ovarian cysts, abnormal fetal presentation and abnormalities of the maternal pelvis are most common risk factors (4). Some studies have proposed that certain body movements might trigger the rotation of the uterus (5). In cases where no risk factors were present, previous caesarean section was found to be a risk factor. In our case there are two risk factors; the presence of uterine leiomyoma and history of previous caesarean sections.

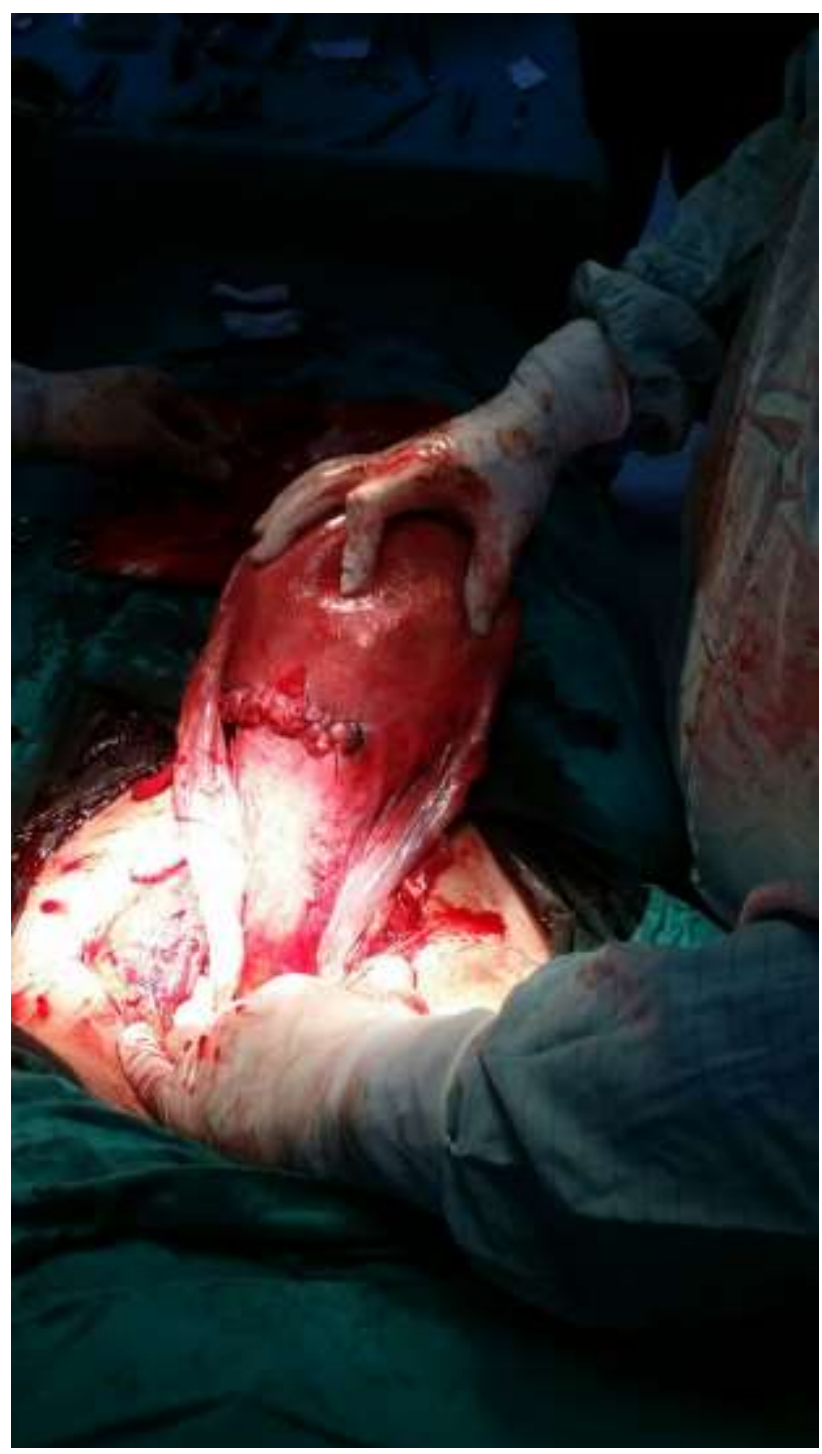

Figure 2: Posterior view of the uterus after detorsion.

Signs and symptoms of the uterine torsion are usually nonspecific. The most common symptom is abdominal pain that varies from mild discomfort to acute abdomen with shock. Other signs and symptoms may include abnormal fetal heart rate, obstructed labor, vaginal bleeding, urinary or intestinal complaints. ${ }^{6}$ In our case hematuria was the only symptom.

It is very difficult to diagnose this rare occasion antenatally due to the nonspecific clinical manifestations and possible asymptomatic cases. The change of placental site compared to the previous ultrasound scans and the abnormal position of ovarian vessels across the uterus on Doppler ultrasound for diagnosis. MRI shows $\mathrm{X}$-shaped configuration of the upper vagina. ${ }^{7}$ On the contrary, ultrasound findings are inconclusive and diagnosis is almost always made intraoperatively like in our case. 
Anterior uterine incision should be made after correcting torsion. In most of the cases derotation is not possible. If derotation is not possible, a posterior low transverse incision should be given. ${ }^{8}$ Risk of rupture of the posterior lower segment incision in next pregnancy is not known. There are reports of myomectomy and posterior vertical incisions in some cases. ${ }^{9,10}$ To prevent recurrence of the torsion plication of round ligaments or uterosacrals are reported in the literature. ${ }^{11,12}$ In our case no recurrence of the torsion was seen in the follow-up period.

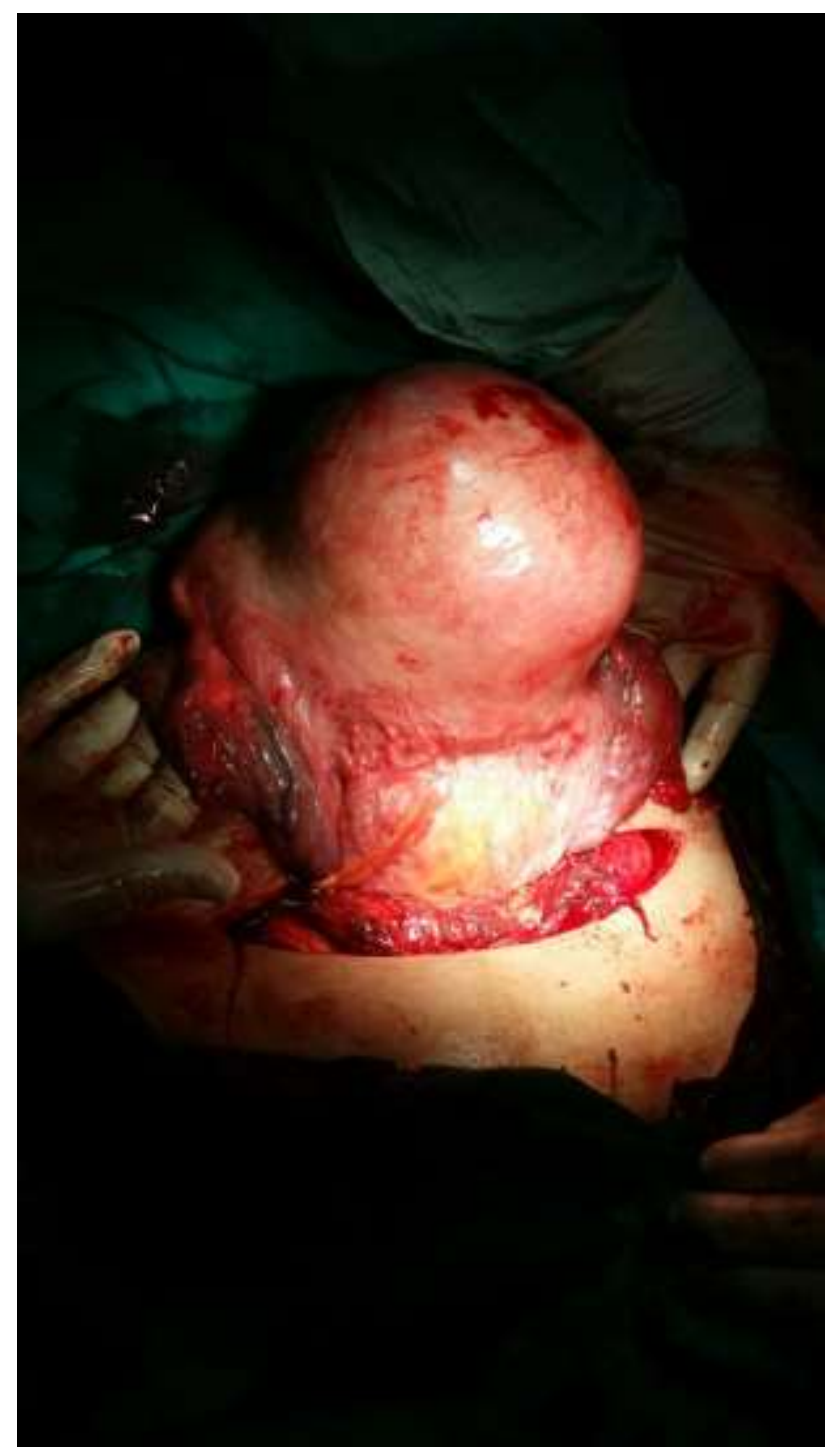

Figure 3: Anterior view of the uterus after detorsion.

\section{CONCLUSION}

Although uterine torsion in pregnancy is very rare accurate treatment is very important hence it can result in severe complications like maternal and neonatal mortality. Posterior low transverse hysterotomy can be made if detorsion of the uterus is not feasible.

\section{Funding: No funding sources \\ Conflict of interest: None declared \\ Ethical approval: Not required}

\section{REFERENCES}

1. Piot D, Gluck M. Torsion of gravid uterus. The Canadian Medical Association Journal. 1973;109(10):1010-1.

2. Zullino S, Faiola S, Paganelli AM, Ferrazzi E. A Case of Abruptio Placentae due to the Torsion of Gravid Uterus. Case Rep Obstet Gynecol. 2014:801616.

3. Wilson D, Mahalingham A. Third trimester uterine torsion: case report. Journal of Obstetrics and Gynaecology Canada. 2006;28(6):531-5.

4. Jensen JG. Uterine torsion in pregnancy. Acta Obstetriciaet Gynecologica Scandinavica. 1992;71(4):260-5.

5. Robinson AL, Duvall HM. Torsion of the pregnant uterus. J Obstet Gynaec Br Common. 1931;38:55-84.

6. Homam H, Moukhah S. Asymptomatic torsion of a gravid uterus. Taiwanese Journal of Obstetrics and Gynecology. 2013;52(4):599-601.

7. Nicholson WK, Coulson CC, McCoy MC, Semelka RC. Pelvic magnetic resonance imaging in the evaluation of uterine torsion. Obstetrics and Gynecology. 1995;85(5, part 2):888-90.

8. Albayrak M, Benian A, Ozdemir I, Demiraran Y, Guralp O. Deliberate posterior low transverse incision at cesarean section of a gravid uterus in 180 degrees of torsion: a case report. J Reprod Med. 2011;56(3-4):1813 .

9. Bolaji II, Rafla NM, Mylotte MJ. Classical caesarean section through the posterior uterine wall. The Irish Journal of Medical Science. 1992;161(2):46-7.

10. Deshpande G, Kaul R, Manjuladevi P. A Case of Torsion of Gravid Uterus Caused by Leiomyoma. Case Reports in Obstetrics and Gynecology. 2011: 206418.

11. Sparic R, Pervulov M, Stefanovic A, Milicevic S. Uterine torsion in term pregnancy. Srp Arh Celok Lek. 2007;135(9-10):572-5.

12. Mustafa MS, Shakeel F, Sporrong B. Extreme torsion of the pregnant uterus. Australian and New Zealand Journal of Obstetrics and Gynaecology. 1999;39(3):360-3.

Cite this article as: Çetin BA, Bahat PY, Dikmen S, Şimşek E, Aydın A. Torsion of term gravid uterus with a large leiomyoma. Int J Reprod Contracept Obstet Gynecol 2017;6:284-6. 